\title{
Evaluation of Public Transport-Based Accessibility to Health Facilities considering Spatial Heterogeneity
}

\author{
Yuliang Zhang $\mathbb{D}^{1}{ }^{1}$ Wenxiang $\mathrm{Li}\left(\mathbb{D},{ }^{2}\right.$ Haopeng Deng, ${ }^{3}$ and $\mathrm{Ye} \mathrm{Li}^{1}$ \\ ${ }^{1}$ The Key Laboratory of Road and Traffic Engineering, Ministry of Education, Tongji University, Shanghai 201804, China \\ ${ }^{2}$ Business School, University of Shanghai for Science and Technology, Shanghai 200093, China \\ ${ }^{3}$ State High-Tech Industrial Innovation Center, Shenzhen 518063, China \\ Correspondence should be addressed to Wenxiang Li; lwxffff@gmail.com
}

Received 5 November 2019; Revised 3 January 2020; Accepted 8 January 2020; Published 1 February 2020

Guest Editor: Tao Liu

Copyright (c) 2020 Yuliang Zhang et al. This is an open access article distributed under the Creative Commons Attribution License, which permits unrestricted use, distribution, and reproduction in any medium, provided the original work is properly cited.

Ensuring adequate public transport-based accessibility to health facilities in different regions is a major concern of social equity and public health for government. However, the imbalanced spatial distribution of health facilities may lead to an inaccurate evaluation of the accessibility, which is shaped by both land use and transportation. To address this problem, this study proposed a new approach to evaluate the adequacy of public transport-based accessibility to health facilities considering the spatial heterogeneity. First, we obtained the spatial distribution of health facilities based on POI data, calculated the population centroids of census tract-based mobile phone positioning data, and estimated travel times from population centroids to every health facility based on web map services. Second, the public transport-based accessibility to health facilities was measured by the isochrone approach. Then, the spatial heterogeneity of the health facilities was quantified by a spatial proximity index based on the gravity model. At last, a benchmark curve of accessibility vs. spatial proximity was established to evaluate the public transport-based accessibility to health facilities in different areas with spatial heterogeneity. A case study of 218 census tracts in Shanghai was conducted to verify this method. Consequently, we successfully identified the census tracts where the public transport-based accessibility to health facilities is insufficient. It shows that even some census tracts within the central city areas are still short of public transport-based accessibility to health facilities, whereas some tracts in the urban periphery may have adequate public transport-based accessibility even though there are limited health facilities nearby.

\section{Introduction}

Because health and treatment are critical to our daily lives, sufficient accessibility to health facilities should be ensured in different regions and for different groups of people. In addition, as a large portion of the patients are the elderly, the poor and the disabled who do not own a car have to depend on public transport service to get to the hospital. Thus, how to evaluate the public transport-based accessibility to health facilities is an important problem that needs to be addressed.

In fact, the accessibility is shaped by two factors, namely, the land use and transportation [1]. Specifically, land use factor can be regarded as the spatial proximity of the health facilities, whereas the transportation factor is the level of public transport services. Because of the aggregation effects of urban populations and resources, spatial differentiation between central urban areas and suburban areas of the city is an inevitable economic trend, which is also referred to as spatial heterogeneity. Therefore, the land use factor resulting in the imbalanced distribution of health facilities may have a more significant impact on the accessibility than the development of public transport. For example, the regions surrounded by many health facilities may have high public transport-based accessibility to health facilities even though the level of public transport service is poor. On the contrary, the public transport-based accessibility in regions with few health facilities around can never reach a high level even though the public transport system is well developed.

Although the land use factor is not easy to be changed because the construction of health facilities is costly and time 
consuming, improving the public transport system is more realistic. Then how to evaluate and improve the public transport-based accessibility in different regions with spatial heterogeneity? Most previous studies just directly compared the accessibility values of the different regions without consideration of the spatial heterogeneity. However, because the land use factor determines the availability and density of health facilities, it is unreasonable to require the regions with few health facilities around to achieve the same level of accessibility as the regions surrounded by many health facilities.

To address the problem above, this article aims to propose a new approach to evaluate the public transportbased accessibility to health facilities considering the spatial heterogeneity. By exploring the relationship between accessibility and spatial proximity of health facilities, we can establish a benchmark curve for evaluation. As a result, there is a benchmark accessibility corresponding to each value of spatial proximity. It is also regarded as the evaluation standard that the public transport-based accessibility should reach. In this way, we can estimate the gap between the actual accessibility and benchmark accessibility and recognize the geographical areas where the public transport-based accessibility to health facilities is insufficient.

The remainder of this article is organized as follows: Section 2 reviews the literature related to this study. The data including points of interests, mobile phone positioning, and travel time are analyzed in Section 3. Afterward, the methods of public transport-based accessibility measurements, spatial proximity analysis of health facilities, and evaluation model considering spatial heterogeneity are described in Section 4. Section 5 discusses the results and policy implications for the improvement of the accessibility in Shanghai. The final section concludes the highlights and limitations of this study and suggests future directions.

\section{Literature Review}

2.1. Measurements of Accessibility. Accessibility can be defined and measured with different methods [1]. These include well-known definitions such as the ease of reaching any activity area using a specific transport system [2], potential opportunities for interaction [3], the freedom of individuals to decide whether to participate in different activities [4], and the overall benefits provided by a given transport system [5]. According to studies of Baradaran and Ramjerdi [6] and Handy and Niemeier [7], accessibility measures have often been classified into four categories: (1) travel-cost approach, (2) isochrone approach, (3) gravitybased approach, and (4) utility-based approach [8]. A comparison of the four accessibility measures is presented in Table 1. Different measures have their own advantages and disadvantages. Researchers need to select the appropriate approach according to the research interests, objectives, and data acquisition.

Overall, existing research has provided a solid foundation for the measurement of accessibility. Different measures have their own advantages and disadvantages. Therefore, researchers need to choose the appropriate approach according to the research interests, objectives, and data acquisition. Because this article focuses on the evaluation of the public transport-based accessibility to health facilities, this article will only apply one of them rather than propose a new method for the accessibility measurement.

2.2. Accessibility to Health Facilities. In terms of accessibility to health facilities, there are many empirical studies in the international literature. Luo and Wang [10] used both the floating catchment area (FCA) method and gravity-based method to examine the spatial accessibility to primary health care in the Chicago ten-county region. They further proposed an enhanced two-step floating catchment area (E2SFCA) method for measuring spatial accessibility to primary care physicians in northern Illinois [11]. These methods can identify persons with inadequate access to primary care physicians. Davy et al. [12] built a framework synthesis to analyze the accessibility to primary health-care services for indigenous people. They found that issues relating to the cultural and social determinants of health such as unemployment and low levels of education can influence whether indigenous patients, their families, and communities were able to access health care. Agbenyo et al. [13] presented an overview of geographic accessibility to health care services in rural Ghana using a mixed approach. They found that poor conditions of roads were the major barriers for household's accessibility to the district hospital.

These studies above mainly focus on the car-based accessibility to health facilities. However, many people, such as the elderly, the poor and the disabled, are typically more dependent on public transport to access the healthcare service. Higgs et al. [14] investigated the impact of different modes of travel (car versus bus) on associations between different measures of General Practitioner (GP) supply and the percentage of elderly patients. Hou and Jiang [15] analyzed the public transport-based accessibility of residential districts to the hospitals during the peak and off-peak hours in Changchun and examined the problems of public transport systems. LaMondia et al. [16] undertook a statistical comparison among four commonly applied measures of transit accessibility to healthcare facilities. Their results indicate that different categories of accessibility measures provide drastically different interpretations of accessibility which are not comparable and interchangeable. However, none of the previous studies have ever discussed the relationship between accessibility to health facilities and the public transport services.

\subsection{Evaluation of Public Transport-Based Accessibility.} Accessibility can be evaluated from various perspectives, including a particular group, mode, location or activity [17]. How accessibility is evaluated affects many planning decisions [18]. Meanwhile, four types of components can be identified from different evaluations: land use, transportation, temporal and individual [1]. In this study, we focus on the public transport-based accessibility, which describes the accessibility of locations to specific destinations by public transport mode. This is different from the public 
TABLE 1: Comparisons of the four accessibility measures [9].

\begin{tabular}{|c|c|c|}
\hline Accessibility & Description & Formula \\
\hline
\end{tabular}

$\begin{array}{llc} & \begin{array}{l}\text { The ease with which any } \\ \text { land-use activity can be } \\ \text { reached from a location } \\ \begin{array}{l}\text { Travel-cost } \\ \text { approach }\end{array}\end{array} \quad A_{i}=\sum_{j=1}^{n}\left(1 / f\left(c_{i j}\right)\right) & \begin{array}{c}\text { (i) Quite easy to } \\ \text { understand and to } \\ \text { calculate }\end{array} \\ & \begin{array}{c}\text { (ii) Less demanding on } \\ \text { data }\end{array}\end{array}$

(i) Neglects variations across individuals

(ii) Highly sensitive to the choice of demarcation area

(iii) Neglects variations in the locations

(i) Neglects variations across individuals

The number of

Isochrone approach opportunities that can be reached within a given travel time, distance, or generalized cost

$$
A_{i}=\sum_{j \in\left\{c_{i j}<c^{*}\right\}} N_{j}
$$

(i) Relatively easy to understand and to calculate

(ii) Easy to visualize (ii) Highly sensitive to the size of the range and the representation of opportunities

(iii) Neglects variations in the locations

(i) Able to differentiate between locations

$\begin{array}{lcc}\text { Gravity-based } & \begin{array}{c}\text { Potential of opportunities } \\ \text { approach }\end{array} & \text { for interaction }\end{array} A_{i}=\sum_{j=1}^{n} N_{j} / f\left(c_{i j}\right)$

(ii) Represents the joint effect of transport systems and land use patterns on accessibility

(i) Neglects variations across individuals

(ii) Ambiguity regarding the magnitude of indicators

(i) Captures individual differences

(ii) Captures the impact (i) Demands extensive data on locations and individuals' travel behavior
Utility-basec
approach
Expected maximum utility considering individual characteristics of all modes including auto, transit, and

nonmotorized options

$$
A_{i}^{n}=\max \left(U_{i j}^{n}\right)=(1 / \mu) \ln \sum_{j=1}^{n} e^{\mu\left(v_{j}^{n}-c_{i j}^{n}\right)}
$$

Note: $A_{i}$ represents the measure of accessibility at location $i, n$ represents the number of included locations, $f\left(c_{i j}\right)$ represents the deterrence function and $c_{i j}$ represents a variable that represents travel cost between locations $i$ and $j, c^{*} c *$ represents the predetermined threshold within which the activity opportunities are counted, $N_{j}$ represents the opportunities in a zone $j, U_{i j}^{n}$ represents the utility of group $n$ selecting the alternative, $v_{j}$ represents some measure reflecting the attraction of the alternative $j$, and $\mu$ represents a positive scale parameter.

transport accessibility which was defined as "the quality of transit serving a particular location and the ease with which people can access that service" [19]. In short, the former is accessibility by public transport mode, while the latter is accessibility to public transport services.

On the one hand, there is a lot of research assessing the accessibility to public transport with consideration of the access to bus stops, duration of public transport journey, and access to destinations via public transport [20]. Many evaluation indexes have been proposed. One of the most recent developments and now seemingly one of the most widely used indexes is the Public Transport Accessibility Level (PTAL) developed by London Borough of Hammersmith and Fullham [21]. It reflects the walking time from a point of interest to the public transport access points, the reliability of the service nodes available, the number of services available within the catchment, and the average waiting time [19].

On the other hand, there are few studies that pay attention to the evaluation of public transport-based accessibility. Majority of them are limited to the simple comparison of accessibility measures. For example, public transport-based accessibility has been compared with carbased accessibility to employment and other land uses in some researches [22-26]. Yan-yan et al. [27] compared the public transport-based accessibility of different traffic analysis zones in Beijing by calculating the accessibility measures based on GIS. In their conclusions, the accessibility in dense-resource regions is always higher than that in sparse resource area [28]. However, there is a problem that the accessibility evaluation criterion for the regions with different spatial attributes should never be consistent because the amounts of potential opportunities are not comparable. In fact, the regions with high accessibility measures not always have sufficient accessibility because the evaluation standard should be higher with more resources to be connected. Therefore, high accessibility measure does not mean sufficient accessibility. These facts may have a great impact on the evaluation result of public transport-based accessibility to health facilities.

In summary, existing studies mainly focus on the measurements of accessibility but rarely look into the adequacy of public transport-based accessibility. Most of them fail to take the spatial heterogeneity into account when directly comparing the accessibility measures in different geographical regions. As a result, it is possible that high accessibility regions still lack public transport services, which cannot be recognized by previous methods. Therefore, it is more reasonable to compare the level of public transport services among the regions with the same spatial proximity 
of the health facilities. In this context, this study expects to improve the evaluation method of public transport-based accessibility to health facilities by incorporating the spatial heterogeneity into the evaluation criterion. In this way, the problem of consistent evaluation criterion for regions with different spatial attributes can be addressed.

\section{Data Preparation}

Shanghai is a megacity covering an area of 6,340.5 square kilometers in China. This study takes Shanghai as a case to evaluate the public transport-based accessibility to health facilities in 218 census tracts, which were defined by the government of Shanghai in 2016 for the purpose of taking a census. We obtained the geography files of the census tracts from the Open Street Map (OSM) to display the boundaries of the census tracts on the map. Besides, the POI data and mobile phone positioning data were collected and analyzed to support this study. Both of them contain the information of the longitudes and latitudes, with which we can match them to the census tracts on the map using the Spatial Analyst Tool "Intersection" of ArcGIS.

3.1. Health Facility Analysis based on POI Data. A point of interest (POI) is a specific point location that someone may find useful or interesting, including businesses, hospitals, hotels, residences, educational buildings, and shopping malls. The POI data are usually described by a name, address, category, and a set of geospatial coordinates [29]. Because this study focuses on the health facilities, the POI data of all hospitals and healthcare centers in Shanghai were collected using the API of Baidu Map which is a web-based tool for interactions between users and enterprises. For example, one of POI data record ("a5097bfab4a5a97af13cbaa3," "Shanghai Changzheng Hospital," "Shanghai," "Huangpu," "415 Fengyang Road," "Medical Facility," “Class 3,” “121.473726," "31.23890086") represents the item ID, name, province, region, address, category tag, class tag, longitude, and latitude, respectively.

As of July 2016, there are 679 regular and authoritative health facilities in Shanghai, including 78 Class-3(best) hospitals (or healthcare centers), 184 Class-2 hospitals, and 417 Class- 1 or other hospitals. Given the addresses and longitudes and latitudes of these health facilities, the spatial distribution is displayed in Figure 1. Because health facilities with different classes may have different attractions for people, it is necessary to consider them separately. In addition, different groups of people may tend to choose different classes of hospitals, which usually depends on the patient's condition, income, age, education, etc. Thus, we give different attraction weights to the three classes of health facilities based on a web-based questionnaire survey where the importance of the three classes of health facilities was rated by 598 respondents sampled randomly. The sample distribution is close to the population distribution. The results show that $56 \%$ respondents tend to choose the Class3 hospital, 29\% respondents tend to choose Class- 2 hospitals, whereas the rest of respondents tend to choose Class- 1 or other hospitals. Then, the weights of different classes are normalized as shown in Table 2.

3.2. Population Distribution Analysis Based on Mobile Phone Positioning Data. As the population is seldom distributed homogeneously within a census tract, the populationweighted centroid rather than the simple geographic centroid of a census tract can represent the location of the population more accurately [10]. The population centroid of a tract may be distant from its geographic centroid, particularly in rural or peripheral suburban areas where tracts are large, and population tends to concentrate in limited space. The geographic coordinate $\left(X_{i}, Y_{i}\right)$ of the population centroid for each census tract $(i)$ is defined as,

$$
\begin{aligned}
& X_{i}=\frac{\sum_{k=1}^{n} P_{k} X_{k}}{\sum_{k=1}^{n} P_{k}}, \\
& Y_{i}=\frac{\sum_{k=1}^{n} P_{k} Y_{k}}{\sum_{k=1}^{n} P_{k}},
\end{aligned}
$$

where $\left(X_{k}, Y_{k}\right)$ is the geographic coordinate of home location $k, P_{k}$ is the resident population of home location $k$, in the census tract $(i)$, and $n$ is the number of distinct home location.

However, the available census data in China can only provide the total population within the census tract, without the exact home locations. Fortunately, lots of studies have proved that mobile phone positioning data can provide good potential to identify the home and work anchor points of the population, since it tracks the positions of every mobile phone whenever physical moving, calling, texting, or surfing the Internet $[9,30]$. Therefore, we use the mobile phone positioning data from China Unicom Communications Corporation of Shanghai collected between March 1, 2016, and March 31, 2016, to infer the home locations of residents.

The dataset contains much useful information such as the mobile identification number of the user, the time when the data record was collected, and the longitude and latitude of the user's estimated location. Then, an algorithm is developed to identify the home locations of residents based on the following assumptions: (1) most people stay at home between 10 pm-6 am every day, (2) the most frequent location appeared during this period is the aggregated home location, and (3) users whose home location remains consistent for more than 20 days during a month are residents of this area. More details about this algorithm could be found in reference $[9,29]$. As a result, the resident population $\left(P_{i}\right)$ of each distinct position $\left(X_{k}, Y_{k}\right)$ can be estimated. Then, the distributions of population densities and centroid of 218 census tracts can be obtained, as shown in Figure 2.

3.3. Travel Time Estimation Based on Web Map Service. There is a tool of Baidu web map services called "Route Matrix API," which can calculate the travel times and distances between origins and destinations in the real-world road network [9]. The input parameters of this tool are 


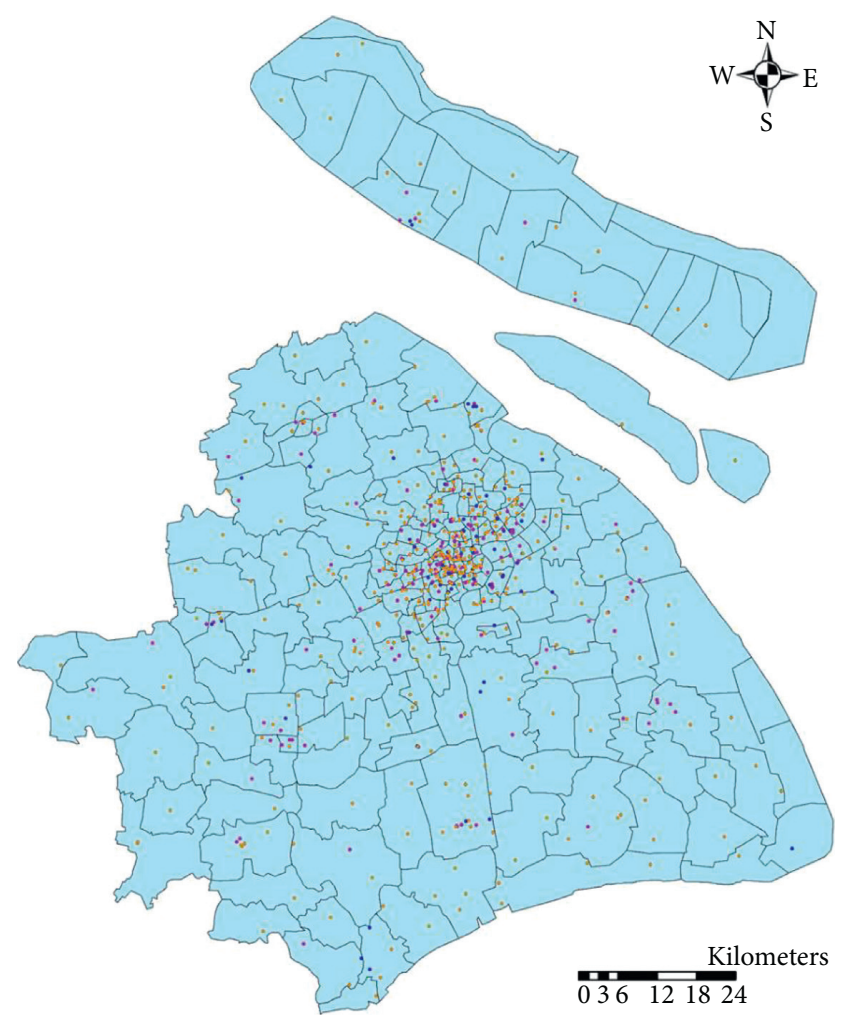

Medical facilities

- Class 3 (78)

- Class 2 (184)

- Class 1 or others (417)

$\square$ Census tract (218)

FIGURE 1: Spatial distribution of health facilities in Shanghai.

TABLE 2: Attraction weights of hospitals with different classes.

\begin{tabular}{lc}
\hline Hospital class & Attraction weight \\
\hline Class 3 & 1 \\
Class 2 & 0.52 \\
Class 1 or others & 0.27 \\
\hline
\end{tabular}

geographic coordinates of the origins and the destinations (health facilities), and the mode of transportation (public transport). It uses the shortest path algorithm to estimate the travel time from origins to destinations by public transport mode considering the real-time and historical traffic status [31]. The estimated travel time also includes walking time from the origin to the public transport station and waiting time at the station. In addition, the public transport data of the web map service are almost complete and up to date, containing all operated routes (of subways, buses, trolley, etc.) in the city. Therefore, the estimated travel times based on this tool are more accurate than the travel times from survey data or planning models [32].

Based on this tool, we can estimate the travel time $\left(t_{i j}\right)$ from each population centroid of the census tract $(i)$ to each health facility $(j)$ by public transport, given the locations of health facilities and population centroid of every census tract which are obtained above. To eliminate the influence of the commuting traffic, all the travel time was measured during 9:00 am-11:00 am at the off-peak hours.

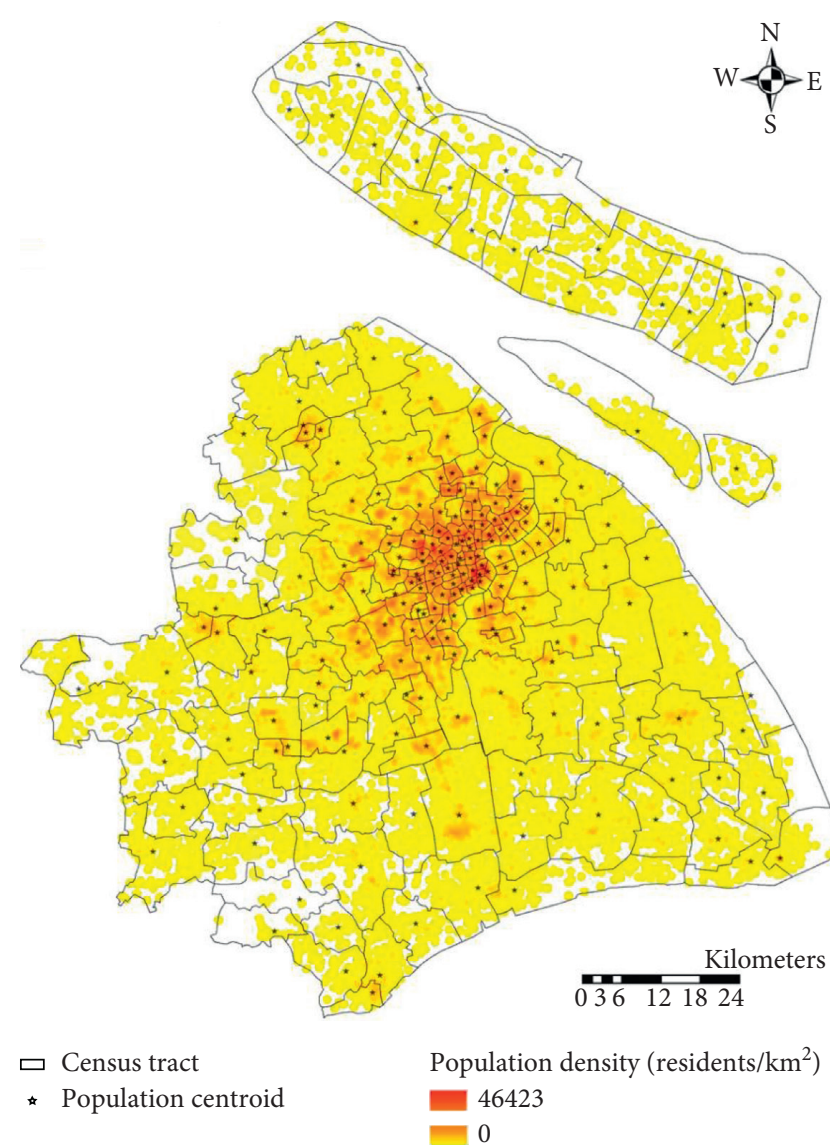

FIgURE 2: Distributions of population densities and centroids of 218 census tracts.

\section{Methodology}

4.1. Measurement of Public Transport-Based Accessibility. This study adopts the isochrone approach which is also referred to as "cumulative opportunity" method to measure the public transport-based accessibility to health facilities. This measurement counts the number of health facilities that can be reached within a given threshold of travel time, distance, or generalized cost by public transport. Specifically, for each census tract $(i)$, search every health facility $(j)$ that are within the threshold travel time $(t)$ from the population centroid of the census tract $(i)$, and sum up the weighted attractions of these health facilities. Mathematically, the public transport-based accessibility to health facilities can be measured as

$$
A_{i}=\sum_{j \in\left\{t_{i j}<t_{0}\right\}} w_{j} N_{j},
$$

where $A_{i}$ represents the public transport-based accessibility for census tract $(i), N_{j}$ represents the total number of attractions at each health facility $(j), w_{j}$ represents the attraction weight of the health facility $(j)$, and $t_{i j}$ represents the travel time from population centroid of census tract $(i)$ to the health facility $(j)$ by public transport. According to the 5 th Shanghai Comprehensive Transport Survey, the average travel time for residents in Shanghai is 29 minutes, whereas 
32.3 minutes is the average travel time in the center area of the city. As a result, we take 30 minutes as the travel time threshold $\left(t_{0}\right)$.

4.2. Spatial Proximity Analysis of Health Facilities. Because the health facilities are distributed unevenly throughout the city, there is inevitably spatial heterogeneity among these census tracts. Meanwhile, the spatial interaction between two geographical entities usually declines as the distance between them increases, which is generally called distance decay [33]. Therefore, these factors may have a great impact on the evaluation of public transport-based accessibility to health facilities. For example, if there are few health facilities available around this census tract, the public transport-based accessibility to health facilities for this tract can never reach a high level, even though the public transport system is good enough. To measure the spatial heterogeneity of the health facilities in different tracts, this study introduces a spatial proximity index derived from the gravity model [34], representing the overall spatial proximity to all available health facilities in the city:

$$
R_{i}=\sum_{j=1}^{m} \frac{w_{j} N_{j}}{d_{i j}^{\eta}},
$$

where $R_{i}$ is the spatial proximity index of health facilities for census tract $(i), m$ is the total number of all health facilities, $d_{i j}$ represents the geodetic distance between the population centroid of census tract $(i)$ and the health facility $(j)$, and $\eta$ represents the distance sensitivity parameter which reflects the degree of unwillingness people feel toward traveling for an extended distance. In this article, we set $\eta=1.08$ according to previous studies [35]. Therefore, we can use the spatial proximity index to represent the spatial heterogeneity, which can also measure the spatial aggregative state of all available health facilities. The larger the $R_{i}$ is, the more concentratedly the health facilities are distributed around the population centroid of the census tract.

4.3. Evaluation of Public Transport-Based Accessibility to Health Facilities. As mentioned above, accessibility is generally determined by two factors: transportation and land use. In this study, these two factors are represented by the level of public transport services and the spatial proximity to all available health facilities respectively. To evaluate the adequacy of public transport services for health facilities independently, it is necessary to eliminate the effects of the spatial proximity. Therefore, we need to construct the relationship between the public transport-based accessibility and the spatial proximity.

Obviously, the accessibility increases with the spatial proximity index if the public transport service is sufficient. However, the growth of the accessibility will not be endless because of the limits of transportation capacity and space (as shown in Figure 3). It is very similar to the growth of population. Consequently, we employ the logistic function which is widely used for population growth [36] to construct the relationship between accessibility and spatial proximity with the following equation:

$$
\widehat{A}_{i}=\frac{\alpha}{1+e^{\left(\beta-R_{i}\right) / \chi}}
$$

where $\widehat{A}_{i}$ is defined as the benchmark accessibility, given a specific value of $R_{i} ; \alpha$ is a parameter representing the growth limit; $\beta$ is a parameter representing $R$ 's value at the inflection point of the curve where the value of $A$ will be $\alpha / 2$; and $\chi$ is a scale parameter.

In general, regression analysis estimates the conditional expectation of the dependent variable, given the independent variables. In other words, it reflects the average value of the dependent variable when the independent variables are fixed [37]. Therefore, the benchmark value $\left(\widehat{A}_{i}\right)$ represents the expected (average) level of accessibility in the census tracts with the same level of spatial proximity. Based on this, we can establish a benchmark curve of accessibility vs. spatial proximity index to identify the census tracts where the public transport services should be improved to match the spatial proximity. If the actual accessibility $\left(A_{i}\right)$ in a census tract is lower than $\widehat{A}_{i}$, then the public transport-based accessibility in that tract is considered to be insufficient, indicating that the public transport services are poor, and vice versa. With the calibrated benchmark model, we can calculate the difference value $\left(\Delta A_{i}\right)$ between the actual accessibility and benchmark accessibility as follows:

$$
\Delta A_{i}=A_{i}-\widehat{A}_{i} \text {. }
$$

However, the absolute value of gap may not suit for comparison. Therefore, we propose an index based on the relative value to evaluate the adequacy of public transportbased accessibility as follows.

$$
\mathrm{EI}_{i}=\frac{\Delta A_{i}}{A_{i}} \times 100 \% \text {. }
$$

This evaluation index represents the percentage by which the actual accessibility is better or worse than the benchmark accessibility [38, 39]. Thus, we can use it to compare and grade the adequacy of public transport-based accessibility of different census tracts with spatial heterogeneity.

\section{Results and Discussions}

With the data and method presented above, we can respectively calculate the public transport-based accessibility to health facilities of 218 census tracts in Shanghai. The spatial distribution of accessibility is presented in Figure 4(a). Overall, the city of Shanghai is divided into 5 regions by 4 main ring roads. The regions within the Outer Ring Road are regarded as the central city areas of Shanghai, while the regions outside the Outer Ring Road are suburban areas. It can be found that the accessibility measures in central city areas are much higher than those of suburban areas. In the central city areas, the closer to the Inner Ring Road, the higher the public transport-based accessibility will be. Besides, the accessibility measures in the west of Huangpu River are usually higher than those in the east of Huangpu River. However, in the suburban areas, the 


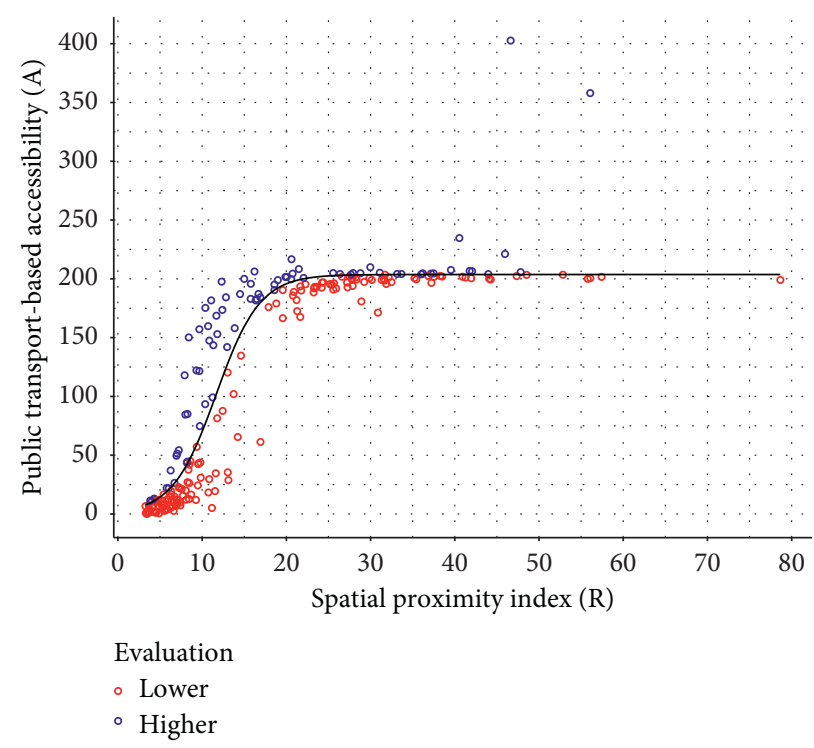

FIGURE 3: Benchmark curve of accessibility vs. spatial proximity.
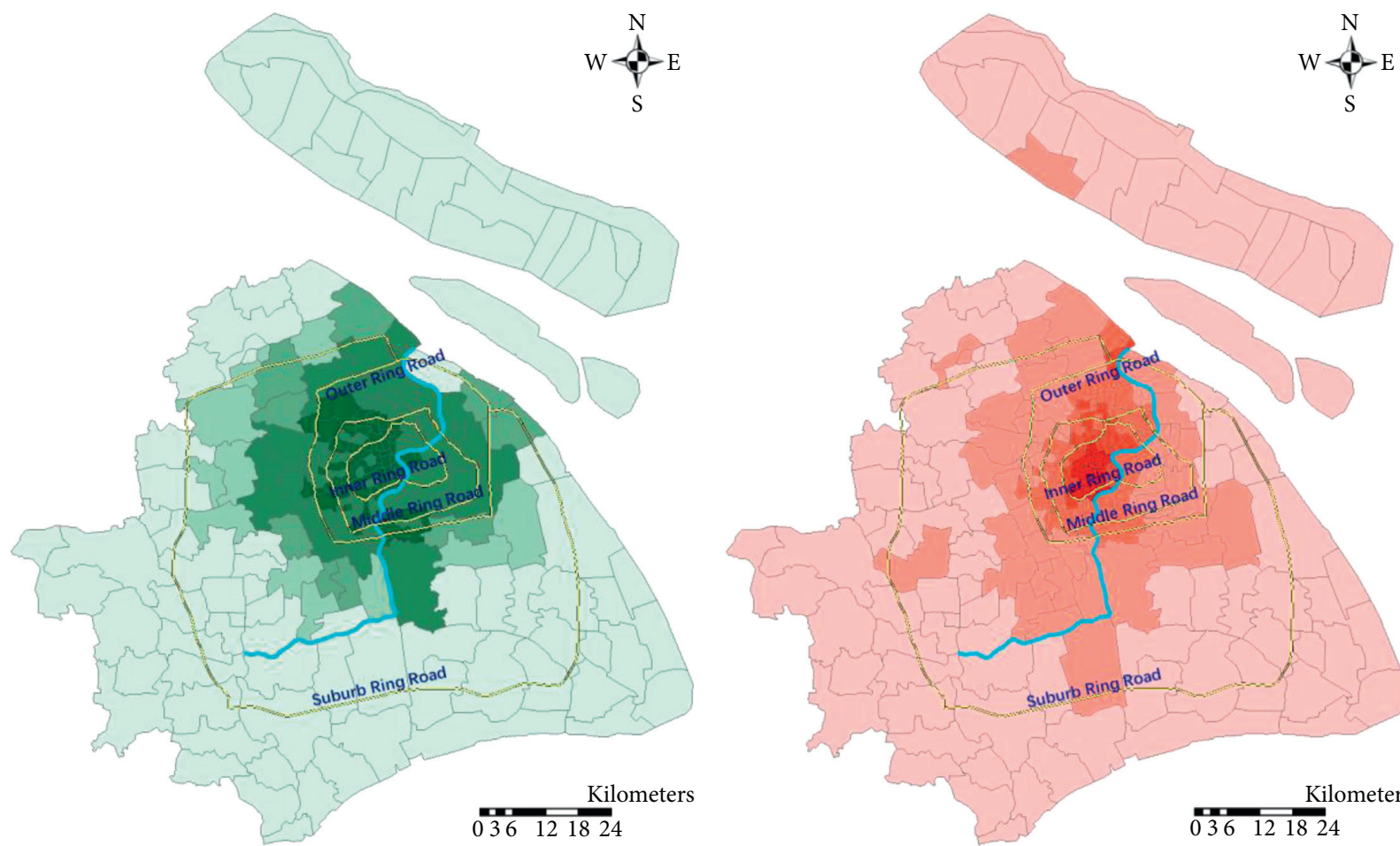

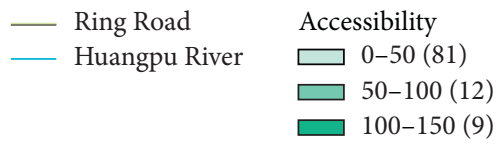

(a)

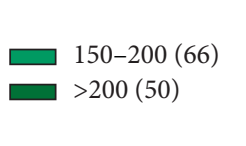
— Ring Road
— Huangpu River

$$
\begin{aligned}
& \square 0-10(85) \\
& \square \text { 10-20(45) } \\
& \square \text { 20-30(44) }
\end{aligned}
$$

Spatial proximity

(b)

FIGURE 4: Spatial distributions of attributes for 218 census tracts. (a) Distribution of public transport-based accessibility. (b) Distribution of spatial proximity to health facilities.

accessibility measures are very low. Given Figure 4(a), we can measure and compare the weighted numbers of health facilities within 30 minutes by public transport in different tracts. However, do the tracts with large numbers of health facilities really have sufficient public transport-based accessibility to health facilities? Whether the short of health 
facilities or the bad public transport services lead to the low accessibility in suburb areas? In fact, we cannot answer these questions by directly comparing the accessibility measures without considering the spatial heterogeneity.

To take the spatial heterogeneity of health facilities into account, we calculate the spatial proximity indexes of the 218 census tracts, respectively, as shown in Figure 4(b), which measures the spatial concentration degree of all available health facilities. The distribution trend of the spatial proximity index is very similar to that of accessibility. It reflects that most health facilities are mainly concentrated in the central city area, especially within the Inner Ring Road. In other words, the census tracts within the Inner Ring Road may have greater potential to access the health facilities if the public transport services are sufficient. Given Figure 4(b), we can only know which tracts are short of health facilities, but we still cannot decide whether the public transport services for health facilities in these tracts are sufficient or not.

To further evaluate the adequacy of public transportbased accessibility to health facilities in different census tracts considering the spatial heterogeneity, we use the accessibility measures and spatial proximity indexes to fit the benchmark curve based on equation (4). The calibration results are listed in Table 3, which indicates that all the estimated parameters are significant, and the function is effective. Thus, the benchmark accessibility can be calculated using this function.

The fitted curve and the actual data points $\left(A_{i}, R_{i}\right)$ of the 218 census tracts are plotted in Figure 3, where the red dots represent the tracts whose accessibility is lower than the benchmark accessibility, whereas the blue ones represent the opposite. If $A_{i}$ is lower than the benchmark accessibility at a fixed value of $R_{i}$, then the accessibility in the census tract ( $\left.i\right)$ is worse than the average level among these tracts with equal spatial proximity. Therefore, we can conclude that the public transport service for health facility in the census $\operatorname{tract}(i)$ should be improved.

Furthermore, we present the relative evaluation index $\left(E_{i}\right)$ of the 218 census tracts, respectively, on the map for comparison, as shown in Figure 5. The census tracts in cold colors (blue) may not have sufficient public transport-based accessibility to health facilities. It shows that above half of 218 census tracts cannot reach the benchmark accessibility. To better understand the results, the subway network which has been built in Shanghai and the bus stops are also plotted in Figure 5. We can find that the tracts not covered by dense subways or bus stations, especially the areas outside the Suburb Ring Road, usually have insufficient accessibility to the health facilities. Thus, it is urgent for these areas to improve their public transport services. However, even some census tracts within the Inner Ring Road may not have adequate public transport-based accessibility to health facilities. Although there are more facilities around these areas, the public transport services may not match with the high level of spatial proximity. Consequently, improvements to public transport services are also needed in some central city area. Although for some tracts around the Outer Ring Road there are limited health facilities nearby, their public transport services are good enough to connect these
TABLE 3: Calibration results of logistic function.

\begin{tabular}{lcccc}
\hline Parameters & Estimate & Std. error & $t$ value & $\operatorname{Pr}(>|t|)$ \\
\hline$\alpha$ & 203.6747 & 3.7496 & 54.32 & $<2 e-16^{* * *}$ \\
$\beta$ & 11.6744 & 0.3041 & 38.39 & $<2 e-16^{* * *}$ \\
$\chi$ & 2.5914 & 0.2470 & 10.49 & $<2 e-16^{* * *}$ \\
\hline
\end{tabular}

Note: Signif. codes: 0 “***” 0.001 “**” 0.01 “*” 0.05 “.” 0.1 “” 1.

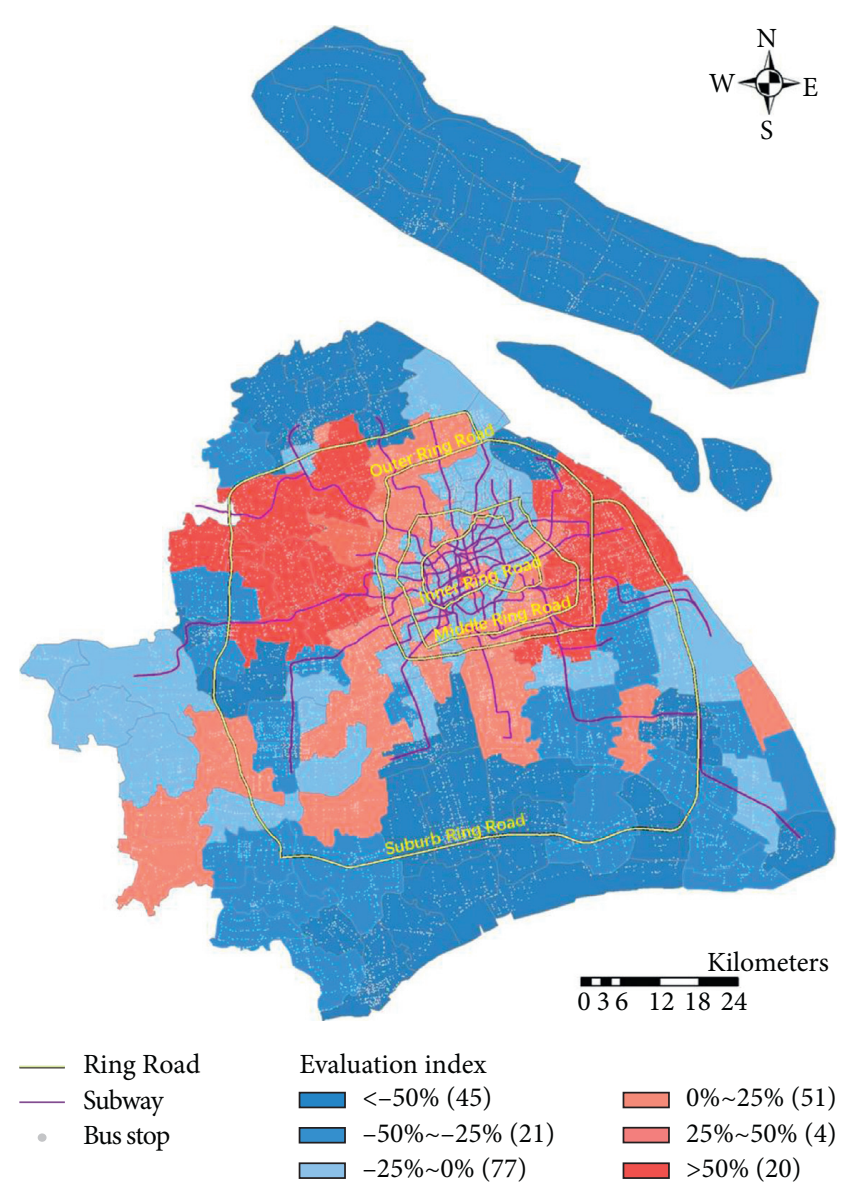

Figure 5: Distribution of evaluation indexes of public transportbased accessibility to health facilities.

available facilities. Therefore, it is not necessary to improve their public transport services. For this condition, only increasing the spatial proximity can further improve their public transport-based accessibility to health facilities.

\section{Conclusion}

This study proposed a novel method to evaluate the adequacy of public transport-based accessibility to health facilities by exploring the relationship between accessibility and spatial proximity of health facilities and establishing a benchmark curve for evaluation criterion. A case study of 218 census tracts in Shanghai was conducted to verify this evaluation method. With a relative evaluation index derived from the benchmark curve, we can compare and grade the adequacy of public transport-based accessibility of different census tracts with different spatial proximity. As a result, we 
successfully identified the census tracts where the public transport-based accessibility to health facilities are insufficient. We find that even some census tracts within the central city areas are still short of public transport-based accessibility to health facilities, whereas some tracts in the urban periphery may have adequate public transport-based accessibility even though there are limited health facilities nearby. However, previous studies mostly failed to recognize these counterintuitive results mainly because of neglecting the spatial heterogeneity in different areas.

To sum up, the primary contribution of this research is to introduce a novel method to evaluate the adequacy of public transport-based accessibility to health facilities by exploring the relationship between accessibility and spatial proximity of health facilities. Specifically, we discover that the growth law of the accessibility vs. spatial proximity can be described by the logistic function, with which a benchmark curve can be established for the accessibility evaluation. In this way, we can efficiently recognize the geographical areas where the public transport-based accessibility to health facilities is insufficient. It is the first time that the effect of public transport on public health has been separated from the accessibility evaluation. Therefore, this research can not only help the government to better evaluate the public transportbased accessibility to health facilities but also provide a clear direction for decision-maker to implement the accessibility and healthcare improvement policies.

However, a few limitations still exist in this study, which also motivates a few future research directions. First, the measurement of accessibility only used a relatively simple and easy method (isochrones approach) because of the data availability. In future studies, a more comprehensive approach to measuring the public transport-based accessibility, which also incorporates the spatial heterogeneity of public facilities, would be proposed. Second, the logistic function is calibrated using only 218 census tracts' data. If the spatial scale of each zone can be made much smaller, the results may be even more accurate. What is more, other public facilities and additional cases should be applied to verify the effectiveness and applicability of this approach.

\section{Data Availability}

The mobile phone data used to support the findings of this study have not been made available because we do not have right to share this to the public. But we can provide part of the encrypted data. The POI data used to support the findings of this study are available from the corresponding author upon request.

\section{Conflicts of Interest}

The authors declare that there are no conflicts of interest regarding the publication of this article.

\section{Acknowledgments}

This study was supported by the projects of the National Natural Science Foundation of China (grant numbers:
71774118 and 91546115) and the project of Science and Technology Commission of Shanghai Municipality (grant number: 16511105204).

\section{References}

[1] K. T. Geurs and B. van Wee, "Accessibility evaluation of landuse and transport strategies: review and research directions," Journal of Transport Geography, vol. 12, no. 2, pp. 127-140, 2004.

[2] M. Q. Dalvi and K. M. Martin, "The measurement of accessibility: some preliminary results," Transportation, vol. 5, no. 1, pp. 17-42, 1976.

[3] W. G. Hansen, "How accessibility shapes land use," Journal of the American Institute of Planners, vol. 25, no. 2, pp. 73-76, 1959.

[4] L. D. Burns, Transportation, Temporal, and Spatial Components of Accessibility, Lexington Books, Lexington, MA, USA, 1979.

[5] M. Ben-Akiva and S R. Lerman, "Disaggregate travel and mobility choice models and measures of accessibility," in Proceedings of the 3rd International Conference on Behavioral Travel Modelling, Tanunda, South Australia, 1979.

[6] S. Baradaran and F. Ramjerdi, "Performance of accessibility measures in europe," Journal of Transportation and Statistics, vol. 4, no. 3, 2001.

[7] S. L. Handy and D. A. Niemeier, "Measuring accessibility: an exploration of issues and alternatives," Environment and Planning A: Economy and Space, vol. 29, no. 7, pp. 1175-1194, 1997.

[8] X. Dong, M. E. Ben-Akiva, J. L. Bowman, and J. L. Walker, "Moving from trip-based to activity-based measures of accessibility," Transportation Research Part A: Policy and Practice, vol. 40, no. 2, pp. 163-180, 2006.

[9] W. Li, Y. Li, X. Ban, H. Deng, H. Shu, and D. Xie, "Exploring the relationships between the non-work trip frequency and accessibility based on mobile phone data," in Proceedings of the 97th Annual Meeting of Transportation Research Board, Washington, DC USA, January 2018.

[10] W. Luo and F. Wang, "Measures of spatial accessibility to health care in a GIS environment: synthesis and a case study in the Chicago region," Environment and Planning B: Planning and Design, vol. 30, no. 6, pp. 865-884, 2003.

[11] W. Luo and Y. Qi, "An enhanced two-step floating catchment area (E2SFCA) method for measuring spatial accessibility to primary care physicians," Health \& Place, vol. 15, no. 4, pp. 1100-1107, 2009.

[12] C. Davy, S. Harfield, A. McArthur, Z. Munn, and A. Brown, "Access to primary health care services for indigenous peoples: a framework synthesis," International Journal for Equity in Health, vol. 15, p. 163, 2016.

[13] F. Agbenyo, A. Marshall Nunbogu, and A. Dongzagla, "Accessibility mapping of health facilities in rural Ghana," Journal of Transport \& Health, vol. 6, pp. 73-83, 2017.

[14] G. Higgs, R. Zahnow, J. Corcoran, M. Langford, and R. Fry, "Modelling spatial access to general practitioner surgeries: does public transport availability matter?" Journal of Transport \& Health, vol. 6, pp. 143-154, 2017.

[15] S. Hou and H. Jiang, "An analysis on accessibility of hospitals in Changchun based on urban public transportation, an analysis on accessibility of hospitals in Changchun based on urban public transportation," Geographical Research, vol. 33, no. 5, pp. 915-925, 2014. 
[16] J. J. LaMondia, C. E. Blackmar, and C. R. Bhat, "Comparing transit accessibility measures: a case study of access to healthcare facilities," in Proceedings of the 90th Annual Meeting of Transport Research Board, Washington, DC, USA, 2011.

[17] T. A. Litman, Evaluating Accessibility for Transport Planning: Measuring People's Ability to Reach Desired Goods and Activities, Victoria Transport Policy Institute, Victoria, Canada, 2017.

[18] T. Litman, "Measuring transportation: traffic, mobility and accessibility," Institute of Transportation Engineers Journal, vol. 73, no. 10, pp. 28-32, 2003.

[19] M. A. Joyce, "Proposed methodology for measuring public transport accessibility to employment sites in the auckland CBD," in Proceedings of the 32nd Australasian Transport Research Forum, Auckland, New Zealand, 2009.

[20] S. Mavoa, K. Witten, T. McCreanor, and D. O'Sullivan, “GIS based destination accessibility via public transit and walking in Auckland, New Zealand," Journal of Transport Geography, vol. 20, no. 1, pp. 15-22, 2012.

[21] Transport for London, Measuring Public Transport Accessibility Levels, Transport for London, London, UK, 2010.

[22] I. Benenson, K. Martens, Y. Rofé, and A. Kwartler, "Public transport versus private car GIS-based estimation of accessibility applied to the tel aviv metropolitan area," The Annals of Regional Science, vol. 47, no. 3, pp. 499-515, 2011.

[23] E. Blumenberg and P. Ong, "Cars, buses, and jobs: welfare participants and employment access in los angeles," Transportation Research Record: Journal of the Transportation Research Board, vol. 1756, no. 1, pp. 22-31, 2001.

[24] D. B. Hess, "Access to employment for adults in poverty in the buffalo-niagara region," Urban Studies, vol. 42, no. 7, pp. 1177-1200, 2005.

[25] M. Kawabata and Q. Shen, "Commuting inequality between cars and public transit: the case of the san francisco bay area, 1990-2000," Urban Studies, vol. 44, no. 9, pp. 1759-1780, 2007.

[26] M. Kawabata and Q. Shen, "Job accessibility as an indicator of auto-oriented urban structure: a comparison of boston and los angeles with tokyo," Environment and Planning B: Planning and Design, vol. 33, no. 1, pp. 115-130, 2006.

[27] C. Yan-yan, W. Pan-yi, L. Jian-hui, F. Guo-chen, L. Xin, and G. Yi, "An evaluating method of public transit accessibility for urban areas based on GIS," Procedia Engineering, vol. 137, pp. 132-140, 2016.

[28] I. Minocha, P. Sriraj, P. Metaxatos, and P. Thakuriah, "Analysis of transit quality of service and employment accessibility for the greater Chicago, Illinois, Region," Transportation research record: Journal of the Transportation Research Board, vol. 2042, pp. 20-29, 2008.

[29] W. Li, Y. Li, J. Fan, and H. Deng, "Siting of carsharing stations based on spatial multi-criteria evaluation: a case study of Shanghai EVCARD," Sustainability, vol. 9, no. 1, p. 152, 2017.

[30] C. Chen, L. Bian, and J. Ma, "From traces to trajectories: how well can we guess activity locations from mobile phone traces?" Transportation Research Part C: Emerging Technologies, vol. 46, pp. 326-337, 2014.

[31] Z. Yao, T. Xu, Y. Chen, Y. Jiang, and B. Ran, "Dynamic platoon dispersion model based on real-time link travel time," IET Intelligent Transport Systems, vol. 13, no. 11, pp. 16941700, 2019.

[32] W. Li, Z. Pu, Y. Li, and X. Ban, "Characterization of ridesplitting based on observed data: a case study of Chengdu,
China," Transportation Research Part C: Emerging Technologies, vol. 100, pp. 330-353, 2019.

[33] P. J. Taylor and S. Openshaw, "Distance decay in spatial interactions," in Proceedings of the Concepts and Techniques in Modern Geography, Norwich, UK, 1975.

[34] A. S. A. Fotheringham, "New set of spatial-interaction models: the theory of competing destinations," Environment and Planning A: Economy and Space, vol. 15, no. 1, pp. 15-36, 1983.

[35] Y. Liu, C. Kang, S. Gao, Y. Xiao, and Y. Tian, "Understanding intra-urban trip patterns from taxi trajectory data," Journal of Geographical Systems, vol. 14, no. 4, pp. 463-483, 2012.

[36] A. Tsoularis and J. Wallace, "Analysis of logistic growth models," Mathematical Biosciences, vol. 179, no. 1, pp. 21-55, 2002.

[37] W. Li, L. Bao, L. Wang, Y. Li, and X. Mai, "Comparative evaluation of global low-carbon urban transport," Technological Forecasting and Social Change, vol. 143, pp. 14-26, 2019.

[38] Z. Yao, L. Shen, R. Liu, Y. Jiang, and X. Yang, "A dynamic predictive traffic signal control framework in a cross-sectional vehicle infrastructure integration environment," IEEE Transactions on Intelligent Transportation Systems, pp. 1-12, 2019.

[39] Z. Yao, Y. Jiang, B. Zhao, X. Luo, and B. Peng, "A dynamic optimization method for adaptive signal control in a connected vehicle environment," Journal of Intelligent Transportation Systems, pp. 1-17, 2019. 